\title{
El clima laboral y la satisfacción laboral en el desempeño docente de instituciones educativas públicas
}

\section{The labor climate and job satisfaction in the teaching performance of public educational institutions}

\author{
Dra. Delsi Mariela Huaita Acha. \\ Dr. Freddy Felipe Luza Castillo.
}

Universidad César Vallejo, Perú

Autor para correspondencia: delsihuaita@gmail.com, freddyluzac@gmail.com

Fecha de recepción: 20 de julio de 2018 - Fecha de aceptación: 30 de agosto de 2018

Resumen: La investigación tuvo el objetivo de determinar la influencia del clima laboral y la satisfacción laboral en el desempeño docente de las instituciones educativas del nivel secundaria de Barrios Altos-Lima. La investigación fue de tipo básica, explicativa y descriptiva. El diseño no experimental, correlacional causal y de corte transversal, se pretendió analizar relaciones causales entre variables a partir de planteamientos de hipótesis causales. El muestreo fue probabilístico, siendo el tamaño de muestra 103 docentes de instituciones educativas públicas del distrito de Barrios Altos. Para observar las variables se empleó tres cuestionarios: Cuestionario de clima laboral, cuestionario de satisfacción laboral y cuestionario de desempeño docente, los cuales fueron validados en su contenido. La confiabilidad fue calculada mediante el coeficiente alfa de Cronbach siendo los resultados 0,79; 0,87 y 0,86 respectivamente. El nivel de predicción de las variables clima laboral y satisfacción laboral juntas sobre el desempeño docente es a un nivel estadístico significativo $\left(\mathrm{x}^{2}=115,746 ; \mathrm{gl}=16 ; \mathrm{p}=0.000\right)$; además, el valor $\mathrm{R}^{2}$ de Nagelkerke obtenido indica que el modelo propuesto explica el $86,6 \%$ de la variable dependiente $(0.866)$.

Palabras Claves: clima laboral; satisfacción laboral; desempeño docente; mejora de los aprendizajes

Abstract: The research had the objective of determining the influence of the labor climate and job satisfaction in the teaching performance of the educational institutions of the secondary level of Barrios Altos-Lima. The research was basic, explanatory and descriptive. The non-experimental, causal correlational and cross-sectional design was used to analyze causal relationships between variables based on the hypothesis of causal hypotheses. Sampling was probabilistic, with the sample size being 103 teachers from public educational institutions in the district of Barrios Altos. To observe the variables, three questionnaires were used: Work climate questionnaire, job satisfaction questionnaire and teacher performance questionnaire, which were validated in their content through expert judgment. Reliability was calculated using Cronbach's alpha coefficient, with the results being $0.79,0.87$, and 0.86 , respectively. The prediction level of the variables labor climate and job satisfaction together on teacher performance is at a significant statistical level (x2 $=115,746, \mathrm{gl}=16, \mathrm{p}=0.000$ ); In addition, the Nagelkerke $\mathrm{R} 2$ value obtained indicates that the proposed model explains $86.6 \%$ of the dependent variable $(0.866)$.

Key Words: labor climate; job satisfaction; teacher performance; improvement of learning 


\section{Introducción}

El primer componente, para alcanzar la calidad de los servicios ofertados en una institución educativa está directamente relacionado con el nivel de satisfacción de los profesionales que trabajan en él y ello generalmente pasa por una valoración del clima laboral que se percibe dentro. Es el caso de las instituciones educativas de Barrios Altos donde los docentes no se hallan satisfechos en su espacio laboral ya que perciben un clima institucional adverso determinado por la brecha de desigualdad en cuanto a capacidades, remuneración y compromisos para su institución; lo que en el tiempo afecta su desempeño; siendo los estudiantes los principales perjudicados.

Con el estudio se pretendió dar mayor luz en cuanto a la comprensión de los factores causales del desempeño docente, sobre todo desde la perspectiva del clima y la satisfacción laboral pues son los principales referentes de un óptimo o pésimo desempeño.

Al revisar diversos antecedentes se pudo evidenciar estudios realizados en universidades, empresas, instituciones educativas que relacionaron en sus resultados directamente las variables dependientes. La investigación resultó relevante ya que determinó la causalidad entre las variables de estudio y aportó información que será el punto de partida para nuevas investigaciones sobre la mejora de los aprendizajes, facilitando una educación de calidad que coadyuve al mejoramiento del logro de competencias preparando para la vida a los futuros ciudadanos. Por otro lado, brinda insumos a tomar en cuenta por los directivos de instituciones educativas públicas para mejorar el desempeño de sus docentes a través de esfuerzos planificados para mejorar su clima laboral.

En búsqueda de mejorar el rendimiento de los estudiantes peruanos se identificó el problema ¿Qué influencia tienen el clima laboral y la satisfacción laboral en el desempeño docente de las instituciones educativas del nivel secundaria de Barrios Altos-Lima, año 2017 ? Cuyos resultados y análisis contribuyeron significativamente en la toma de decisiones de los directivos de los colegios encuestados ya que el propósito de la investigación fue básicamente mejorar los aprendizajes de dicha población.

\section{Hipótesis}

Hipótesis general:

El clima laboral y la satisfacción laboral influyen significativamente en el desempeño de los docentes que laboran en instituciones educativas del nivel secundarias de Barrios Altos-Lima.

Hipótesis específicas:

El clima laboral y la satisfacción laboral influyen significativamente en la preparación para el aprendizaje de los docentes que laboran en instituciones educativas del nivel secundarias de Barrios Altos-Lima. 
El clima laboral y la satisfacción laboral influyen significativamente en la enseñanza para el aprendizaje de los docentes que laboran en instituciones educativas del nivel secundarias de Barrios Altos-Lima.

El clima laboral y la satisfacción laboral influyen significativamente en la participación de la gestión de los docentes que laboran en instituciones educativas del nivel secundarias de Barrios Altos-Lima

El clima laboral y la satisfacción laboral influyen significativamente en la profesionalidad e identidad de los docentes que laboran en instituciones educativas del nivel secundarias de Barrios Altos-Lima.

Al plantear las hipótesis correlaciónales causales se pretendió describir la relación entre las variables, sus dimensiones y las posibles causas en la búsqueda de posibles explicaciones esto implicó que si un docente no está satisfecho con su trabajo o con el clima existente en el mismo entonces su rendimiento no será el óptimo.

Esto lleva a inferir que aquellos aspectos vinculados al ambiente de trabajo relacionados al funcionamiento de procesos y optimización de resultados (Palma, 2004), hace que los docentes propicien aprendizajes de manera reflexiva, critica y creativa con sus estudiantes (MINEDU, 2012). El ministerio de Educación peruano tuvo una nueva visión de mejora de la educación ya que debe realizar acciones para garantizar que los maestros gocen de un clima laboral adecuado y se sientan satisfechos con el trabajo que realizan fomentando de esta manera un mejor desempeño de los mismos.

\section{Métodos}

El tipo de investigación es básica y nivel explicativo porque "va más allá de la descripción de conceptos o fenómenos, así como el establecimiento de relaciones entre conceptos. Están dirigidos a responder por las causas de los eventos y fenómenos físicos o sociales" (Valderrama, 2013, p. 173). La muestra fue de tipo probabilística compuesta por 103 docentes de instituciones educativas del nivel secundaria de Barrios Altos-Lima. La técnica que se utilizó fue la encuesta y el instrumento de recolección de datos fue el cuestionario, a lo que los encuestados responden en una escala tipo Likert. Para la validez de los instrumentos se utilizó el juicio de expertos y para la confiabilidad del instrumento se utilizó el Coeficiente Alfa de Cronbach.

Los datos fueron obtenidos en forma colectiva, contando con el consentimiento informado respectivo. El análisis descriptivo es presentado en tablas de frecuencias y porcentajes y la parte inferencial, que corresponde a la comprobación de hipótesis, se realizaron mediante regresión logística.

\section{Característica del participante}

Las variables fueron medidas por dos instrumentos validados por la psicóloga organizacional Sonia Palma (2005), el clima laboral y la satisfacción laboral el tercer 
instrumento desempeño docente fue elaboración propia sometido a juicio de expertos y con un análisis de confiabilidad de 0,86 con lo que se concluyó que el instrumento es confiable.

La muestra fue tomada por única vez, se utilizó el programa estadístico SPSS versión 21, la muestra fue determinada por la fórmula de (Cochran, 1990) seleccionando en total 81 docentes de cuatro instituciones educativas del Cercado de Lima.

El muestreo fue aleatorio probabilístico, la técnica empleada fue la encuesta

\section{La primera variable es el clima laboral}

Al hablar de clima laboral podemos pensar en la percepción propia de cada miembro de la institución y si las juntamos y las hacemos interactuar surge el clima o microclima de cada uno de los ambientes de la empresa que giran en torno a las experiencias de cada uno, matizado por las actividades que se presenten. Cada persona constituye un micro mundo y el todo es la empresa.

\section{Dimensiones del clima laboral}

Según La Escala CL-SPC de medición del clima laboral, diseñada y elaborada por Palma (2004), cuyas dimensiones son:

Autorrealización. Según Palma (2004), Autorrealización es la percepción del empleado acerca de las oportunidades que ofrece la institución para desarrollarse personal y profesionalmente en función a la labor que se realiza.

Involucramiento laboral. Según Palma (2004), el compromiso es esencial para la conformación de equipos de trabajo eficiente y eficaz. El compromiso primero se desarrolla en una persona y luego fluye en los demás, y está determinado por los objetivos comunes, por ésta razón se considera importante a la dimensión para la investigación a realizar.

Supervisión. Según Palma (2004), son las apreciaciones de funcionalidad y significación de la supervisión de los superiores durante la actividad laboral en tanto signifiquen apoyo u orientación para mejorar el trabajo. Son ejemplos de esta área: a) El supervisor brinda apoyo para superar los obstáculos que se presentan; b) La evaluación que se hace del trabajo ayuda a mejorar.

Comunicación. Según Palma (2004), es la percepción del grado de claridad, coherencia y exactitud de la información que fluye internamente en la institución tanto con los usuarios como entre el personal.

Condiciones laborales. Según Palma (2004), es el esfuerzo que la institución realiza para brindar los insumos materiales, económicos y/o condiciones psicosociales necesarios para que el trabajador cumpla con la labor encomendada.

\section{La segunda variable es la satisfacción laboral}


Para Barraza y Ortega (2009, citado por Jaik, Tena y Villanueva, 2010) definen satisfacción laboral como:

La actitud que muestra el trabajador frente a su trabajo, y que esa actitud se basa en creencias y valores que el trabajador desarrolla de su propio trabajo y que necesariamente influirán de manera significativa en sus comportamientos y en sus resultados (p. 121).

\section{Dimensiones de satisfacción laboral}

Sus dimensiones son las siguientes (Palma, 2005):

Significación tarea. Disposición al trabajo en función a atribuciones asociadas a que el trabajo personal logra sentido de esfuerzo, realización, equidad y/o aporte material.

Condiciones de trabajo. Evaluación del trabajo en función a la presencia o de directivas, normativas, o condiciones que regulen la actividad laboral. Ejemplo de ella sería La comodidad de mi ambiente de trabajo es inigualable.

Reconocimiento personal y/o social. Tendencia evaluativa del trabajo en función al reconocimiento propio o de personas asociadas al trabajo, con respecto a los logros en el trabajo o por el impacto de éstos en resultados indirectos. Ejemplo de ello sería: Siento que recibo de parte de la empresa mal trato.

Beneficios económicos. Disposición al trabajo en función a aspectos remunerativos o incentivos económicos como producto del esfuerzo en la tarea asignada. Ejemplo de ello sería la pregunta: Me siento mal con lo que gano.

La tercera variable es el desempeño docente: Valdés (2006), señala que el desempeño de un profesor es "un proceso sistemático de obtención de datos válidos y fiables, con el objetivo de comprobar y valorar el efecto educativo que produce en los alumnos el despliegue de sus capacidades pedagógicas, su emocionalidad, responsabilidad laboral y la naturaleza de sus relaciones interpersonales con alumnos, padres, directivos, colegas y representantes de las instituciones de la comunidad".

\section{Preparación para el aprendizaje de los estudiantes:}

Comprende la planeación del trabajo pedagógico a través de la elaboración del programa curricular, las unidades didácticas y las sesiones de aprendizaje en el marco de un enfoque intercultural e inclusivo. Refiere el conocimiento de las principales características sociales, culturales, materiales e inmateriales y cognitivas de sus estudiantes, el dominio de los contenidos pedagógicos y disciplinares, así como la selección de materiales educativos, estrategias de enseñanza y evaluación del aprendizaje.

\section{Enseñanza para el aprendizaje de los estudiantes}


Comprende la conducción del proceso de enseñanza por medio de un enfoque que valore la inclusión y la diversidad en todas sus expresiones. Refiere la mediación pedagógica del docente en el desarrollo de un clima favorable al aprendizaje, el manejo de los contenidos, la motivación permanente de sus estudiantes, el desarrollo de diversas estrategias metodológicas y de evaluación, así como la utilización de recursos didácticos pertinentes y relevantes. Incluye el uso de diversos criterios e instrumentos que facilitan la identificación del logro y los desafíos en el proceso de aprendizaje, además de los aspectos de la enseñanza que es preciso mejorar (MINEDU, 2012, p. 20).

\section{Participación en la gestión de la escuela articulada a la comunidad}

El involucramiento de la comunidad en el proceso de enseñanza aprendizaje se hace relevante en medida que la función educadora se amplía en el contexto. En ese sentido la relación con padres y comunidad se hace necesaria (ORELAC-UNESCO, 2007). Por su parte Valdés (2004), menciona que la Naturaleza de las relaciones interpersonales que establece con alumnos, padres, directivos y colegas es facilitadora del aprendizaje.

\section{Desarrollo de la profesionalidad y la identidad docente}

El desarrollo de la profesionalidad está en cierta medida ligado al cumplimiento de normas y la actitud y personalidad del docente (ORELAC-UNESCO, 2007), la responsabilidad, (Manzi, Gonzales y Yulan, 2011), y al compromiso (SEICE, 2011).

Comprende el proceso y las prácticas que caracterizan la formación y desarrollo de la comunidad profesional de docentes. Refiere la reflexión sistemática sobre su práctica pedagógica, la de sus colegas, el trabajo en grupos, la colaboración con sus pares y su participación en actividades de desarrollo profesional. Incluye la responsabilidad en los procesos y resultados del aprendizaje y el manejo de información sobre el diseño e implementación de las políticas educativas a nivel nacional y regional (MINEDU, 2012, p. 20).

Se utilizó el cuestionario sobre clima laboral es un instrumento que consta de 50 ítems con una escala de 5 niveles y su corrección es la suma simple del valor atribuido a cada ítem.

El cuestionario de satisfacción laboral es un instrumento que consta de 27 ítems y responde en una escala de 5 niveles.

El cuestionario de desempeño docente consta de 25 ítems al cual el sujeto responde en una escala de 4 niveles.

\section{Resultados}

\section{Comprobación de la Hipótesis general:}

$\mathrm{H}_{0}$ : El clima laboral y la satisfacción laboral no influyen significativamente en el desempeño de los docentes que laboran en instituciones educativas del nivel secundario de Barrios Altos-Lima. 
$\mathrm{H}_{\mathrm{G}}$ : El clima laboral y la satisfacción laboral influyen significativamente en el desempeño de los docentes que laboran en instituciones educativas del nivel secundario de Barrios AltosLima.

Tabla 1. Estadística de información del modelo: razón de verosimilitud de clima laboral*satisfacción laboral y desempeño docente

\begin{tabular}{|c|c|c|c|c|c|c|}
\hline \multirow[t]{2}{*}{ Modelo } & \multicolumn{3}{|c|}{ Criterio de ajuste del modelo } & \multicolumn{3}{|c|}{$\begin{array}{c}\text { Contrastes de la razón de } \\
\text { verosimilitud }\end{array}$} \\
\hline & AIC & BIC & $\begin{array}{c}-2 \log \\
\text { verosimilitud }\end{array}$ & Chi-cuadrado & $\mathrm{gl}$ & Sig. \\
\hline $\begin{array}{l}\text { Sólo la } \\
\text { intersección }\end{array}$ & 128,833 & 133,622 & 124,833 & & & \\
\hline Final & 45,087 & 88,187 & 9,087 & 115,746 & 16 &, 000 \\
\hline
\end{tabular}

El valor $\mathrm{x}^{2}$ del modelo empírico a la que se aproxima la razón de verosimilitud es de $115,746$ con 16 grados de libertad y un nivel de significancia menor a 0.05 ( $\mathrm{p}=0.000)$, por lo que se rechaza la hipótesis nula; es decir el clima laboral y la satisfacción laboral influyen significativamente en el desempeño de los docentes que laboran en instituciones educativas del nivel secundario de Barrios Altos-Lima.

Tabla 2. Valor $\mathrm{R}^{2}$ de Nagelkerke de clima laboral*satisfacción laboral y desempeño docente

\begin{tabular}{lr}
\hline \multicolumn{2}{l}{ Pseudo R-cuadrado } \\
\hline Cox y Snell &, 760 \\
Nagelkerke &, 866 \\
McFadden &, 680
\end{tabular}

El valor $\mathrm{R}^{2}$ de Nagelkerke indica que el modelo propuesto explica el $86,6 \%$ de la variable dependiente (0.866).

\section{Comprobación de hipótesis especifica 1}

$\mathrm{H}_{0}$ : El clima laboral no influye significativamente en el desempeño de los docentes que laboran en instituciones educativas del nivel secundario de Barrios Altos-Lima.

$\mathrm{H}_{1}$ : El clima laboral influye significativamente en el desempeño de los docentes que laboran en instituciones educativas del nivel secundario de Barrios Altos-Lima.

Tabla 3. Estadística de información del modelo: razón de verosimilitud de clima laboral y desempeño docente

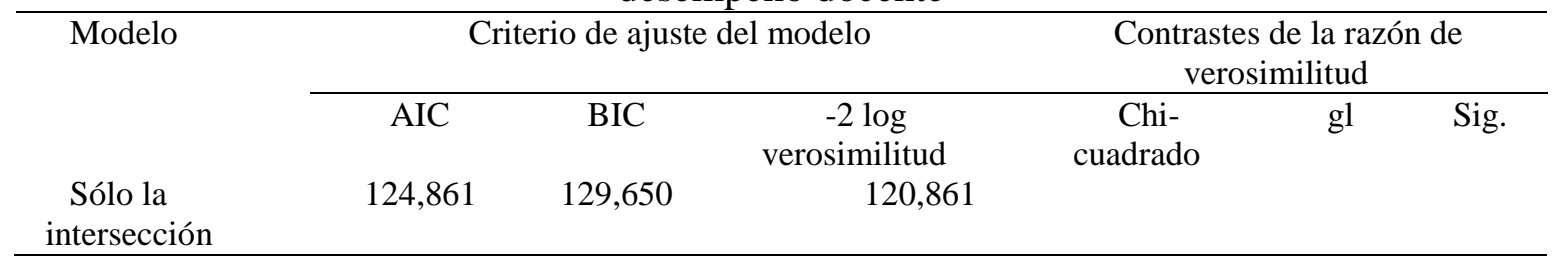




\begin{tabular}{lllllll} 
Final & 29,297 & 53,242 & 9,297 & 111,564 & 8 &, 000 \\
\hline
\end{tabular}

El valor $\mathrm{x}^{2}$ del modelo empírico a la que se aproxima la razón de verosimilitud es de $111,564$ con 8 grados de libertad y un nivel de significancia menor a 0.05 ( $p=0.000)$, por lo que se rechaza la hipótesis nula; es decir el clima laboral influye significativamente en el desempeño de los docentes que laboran en instituciones educativas del nivel secundario de Barrios AltosLima.

Tabla 4. Valor $\mathrm{R}^{2}$ de Nagelkerke de clima laboral y desempeño docente Pseudo R-cuadrado

\begin{tabular}{ll}
\hline Cox y Snell &, 748 \\
Nagelkerke &, 852 \\
McFadden &, 655
\end{tabular}

El valor $\mathrm{R}^{2}$ de Nagelkerke indica que el modelo propuesto explica el 85,2\% de la variable dependiente (0.852).

\section{Comprobación de hipótesis especifica 2}

$\mathrm{H}_{0}$ : La satisfacción laboral no influye significativamente en el desempeño de los docentes que laboran en instituciones educativas del nivel secundario del distrito de Barrios Altos-Lima.

$\mathrm{H}_{2}$ : La satisfacción laboral influye significativamente en el desempeño de los docentes que laboran en instituciones educativas del nivel secundario del distrito de Barrios Altos-Lima.

Tabla 5. Estadística de información del modelo: razón de verosimilitud de satisfacción laboral y

\begin{tabular}{lcccccc}
\hline Modelo & \multicolumn{3}{c}{ Criterio de ajuste del modelo } & \multicolumn{3}{c}{$\begin{array}{c}\text { Contrastes de la razón de } \\
\text { verosimilitud }\end{array}$} \\
\cline { 2 - 6 } & AIC & BIC & $\begin{array}{c}-2 \text { log } \\
\text { verosimilitud }\end{array}$ & Chi-cuadrado & gl & Sig. \\
Sólo la & 109,231 & 114,020 & 105,231 & & \\
$\begin{array}{c}\text { intersección } \\
\text { Final }\end{array}$ & 29,813 & 53,757 & 9,813 & 95,418 & 8 &, 000 \\
\hline
\end{tabular}

El valor $\mathrm{x}^{2}$ del modelo empírico a la que se aproxima la razón de verosimilitud es de $95,418$ con 8 grados de libertad y un nivel de significancia menor a 0.05 ( $\mathrm{p}=0.000)$, por lo que se rechaza la hipótesis nula; es decir la satisfacción.

Tabla 6. Valor $\mathrm{R}^{2}$ de Nagelkerke de satisfacción laboral y desempeño docente

Pseudo R-cuadrado

\begin{tabular}{lr}
\hline Cox y Snell &, 692 \\
Nagelkerke &, 789 \\
McFadden &, 561 \\
\hline
\end{tabular}


El valor $\mathrm{R}^{2}$ de Nagelkerke indica que el modelo propuesto explica el 78,9\% de la variable dependiente (0.789).

\section{Discusión}

En la presente investigación se ha encontrado que el clima laboral y la satisfacción laboral influyen significativamente $(\mathrm{p}<0,05)$ en el desempeño docente. El nivel de predicción de las variables clima laboral y la satisfacción laboral sobre el desempeño docente es al 86,6\% ya que el modelo propuesto explica el $86,6 \%$ de la variable dependiente $\left(\mathrm{R}^{2}\right.$ de Nagelkerke=0.866). En general se puede decir que aquellos aspectos vinculados al ambiente de trabajo relacionados al funcionamiento de procesos y optimización de resultados (Palma, 2004), sumado a la disposición hacia el trabajo basadas en creencias y valores (Palma, 2005), hace que los docentes propicien aprendizajes de manera reflexiva, critica y creativa con sus estudiantes (MINEDU, 2012). Sin embargo, hay que tomar en cuenta que en las instituciones educativas evaluadas hay un clima más o menos estable pero no necesariamente sienten satisfacción hacia su trabajo, aun así, el clima determina un desempeño favorable en bien de los estudiantes.

Estos resultados difieren de lo hallado por Pelaes (2010) ya que sus evidencias señalan que el clima organizacional se relaciona con la satisfacción, sobre todo si se toman en cuenta el estilo de dirección, el sentido de pertenencia, la retribución, la estabilidad, la claridad y coherencia de la dirección y los valores colectivos. En todo caso se puede señalar que el clima laboral es mejor predictor que la satisfacción laboral, sobre todo si se toman en cuenta en su desarrollo aspectos como el reconocimiento, la autoestima el autodesarrollo, la estabilidad emocional y la demanda cognitiva que exige la ejecución del trabajo. (Díaz, 2010). Después de todo, según el autor, las variables de motivación y las demandas cognitivas percibidas en el entorno laboral, son las que juegan un mejor rol a la hora de determinar el nivel de rendimiento laboral. A lo que según Quispe (2014), se debe agregar una positiva percepción de la imagen de la institución donde se labora.

Asimismo, se ha evidenciado que clima laboral influye en el desempeño de los docentes que laboran en instituciones educativas del nivel secundario de Barrios Altos-Lima, año 2016. El nivel de predicción es del 85,2\%, dado que la variable independiente explica el $85,2 \%$ de la variable dependiente $\left(\mathrm{R}^{2}\right.$ de Nagelkerke $\left.=0.852\right)$. Esto lleva a inferir que aquellos aspectos vinculados al ambiente de trabajo relacionados al funcionamiento de procesos y optimización de resultados (Palma, 2004), hace que los docentes propicien aprendizajes de manera reflexiva, critica y creativa con sus estudiantes (MINEDU, 2012).

Estos resultados concuerdan con lo hallado por Baños (2011), quien descubrió que el clima organizacional es una variable que afecta directamente a la percepción de la innovación $\mathrm{y}$, al desarrollo de las competencias esenciales que en suma definen al desempeño laboral. Asimismo, puntualiza que la definición del rol logra compromiso entre el trabajador hacia la institución y este compromiso genera un aporte mayor al desarrollo de las competencias esenciales. En la misma línea, La Torre (2012) señala que las prácticas de recursos humanos orientadas al mejoramiento del compromiso laboral es lo que se relaciona positivamente con el desempeño de los empleados. Además, Toala (2014), manifestó que debe existir una efectiva y 
oportuna comunicación para que mejoren las relaciones interpersonales entre autoridades y servidores públicos ya que ello determina un mejor desempeño laboral.

Asimismo, se ha encontrado que la satisfacción laboral influye en el desempeño de los docentes que laboran en instituciones educativas del nivel secundario de Barrios Altos-Lima, año 2016. El nivel de predicción obtenido es a un nivel estadístico significativo $(\mathrm{p}<0,05)$, dado que la variable independiente (satisfacción laboral), explica el 78.9\% de la variable dependiente $\left(\mathrm{R}^{2}\right.$ de Nagelkerke=0.789). Es decir, los docentes que muestran buena disposición hacia el trabajo basadas en creencias y valores (Palma, 2005), también presentan desempeños que hacen que los estudiantes mejoren sus aprendizajes de manera reflexiva, critica y creativa (MINEDU, 2012).

Es resultados son similares a lo hallado por Campos (2013) ya que, en su caso, las evidencias señalan que la satisfacción laboral es un antecesor predictor, en grado moderado, del desempeño laboral. También Subaldo (2012), señala que las experiencias positivas de los profesores en el ejercicio de la docencia producen satisfacción y conducen al desarrollo y a la realización personal y profesional, que efectivamente influyen en la calidad de la enseñanza y los aprendizajes de los estudiantes. Asimismo, Rodríguez, Retamal, Lizana y Cornejo (2012) evidencia que la satisfacción general resulta ser un predictor significativo del rendimiento y productividad del personal 


\section{Conclusión}

El clima laboral y la satisfacción laboral influyen significativamente $(\mathrm{p}<0,05)$ en el desempeño del docente del nivel secundaria de Barrios Altos-Lima, año 2016. El nivel de predicción de las variables clima laboral y satisfacción laboral sobre el desempeño docente es al $54,2 \%$ ya que las variables independientes explican el $54.2 \%$ de la varianza de la variable dependiente (desempeño docente). Aquellos aspectos vinculados al ambiente de trabajo sumado a la disposición hacia el trabajo basadas en creencias y valores hacen que los docentes propicien aprendizajes de manera reflexiva, crítica y creativa con sus estudiantes.

El clima laboral influye en el desempeño de los docentes del nivel secundario de Barrios Altos-Lima, año 2016. El nivel de predicción es a un nivel estadístico significativo ( $\mathrm{p}<0,05)$, dado que la variable independiente (clima laboral), explica el 50,6\% de la varianza de la variable dependiente (desempeño docente). El ambiente laboral donde se desarrolla un docente permite que propicien mejores aprendizajes en sus estudiantes.

La satisfacción laboral influye en el desempeño de los docentes del nivel secundaria de Barrios Altos-Lima, año 2017. El nivel de predicción obtenido es a un nivel estadístico significativo $(\mathrm{p}<0,05)$, dado que la variable independiente (satisfacción laboral), explican el $53.9 \%$ de la varianza de la variable dependiente (desempeño docente, Es decir, los docentes que muestran buena disposición hacia el trabajo, también presentan desempeños que hacen que los estudiantes mejoren sus aprendizajes. 


\section{Bibliografía}

Baños V. (2011). Competencias esenciales, clima organizacional e innovación como factores de Competitividad empresarial. Tesis de la Universidad Autónoma de Madrid. España.

Campos, C. (2013). Modelo de asociación entre factores de satisfacción y desempeño laboral en docentes universitarios. Tesis doctoral de la Universidad de Montemorelos. México.

Chiang, M.; Botello, C. y Núñez, A. (2007). Clima organizacional y satisfacción laboral en un establecimiento de salud estatal: hospital tipo 1. Theoria, 16(2), pp. 61-76. Disponible en http://www.redalyc.org/pdf/299/29916206.pdf

Cochran, W. (1990). Técnicas de Muestreo. México. Compañía Editorial Continental. (p 49)

Díaz, M. (2010). Predicción del rendimiento laboral a partir de indicadores de motivación, personalidad y percepción de factores psicosociales. Tesis doctoral de la Universidad Complutense de Madrid. España.

Jaik, Tena y Villanueva (2010). Satisfacción Laboral y Compromiso Institucional de los Docentes de Postgrado. Revista Electrónica Diálogos Educativos. Obtenido de file:///C:/Users/Freddy/Downloads/Dialnet-

SatisfaccionLaboralYCompromisoInstitucionalDeLosDo-3294739.pdf

La Torre , F. (2012). La gestión de recursos humanos y el desempeño laboral. España: Tesis doctoral de la Universidad de Valencia.

Manzi, Gonzales y Yulan, (2011). La Evaluación Docente en Chile. Santiago de Chile. Edit. Universidad Católica de Chile.

MINEDU (2012). Marco de Buen Desempeño Docente. Lima: MINEDU.

OREALC/UNESCO. (2007). Evaluación del desempeño y carrera profesional docente. Un estudio comparado entre 50 países de América y Europa. Santiago: UNESCO.

Palma, S. (2004). Escala de Clima Laboral (CL-SPC) Manual. Lima: Editora y Comercializadora CARTOLAN EIRL.

Palma, S. (2005). Escala de Satisfacción Laboral (SL-SPC) Manual. Lima: Editora y Comercializadora CARTOLAN EIRL.

Pelaes, O, (2010). Relación entre el clima organizacional y la satisfacción del cliente en una empresa de servicios telefónicos. Tesis para obtención de grado de Magister. Universidad Nacional Mayor de San Marcos, Perú. 
Quispe D. (2014). Clima laboral y percepción de la imagen institucional en el instituto de educación superior tecnológico público Juan Velasco Alvarado, año 2013. Tesis doctoral de la Universidad de San Martin de Porres. Lima.

Rodriguez, G. (2012). Obtenido de http://ruc.udc.es/dspace/bitstream/2183/5669/1/RodriguezFuentes_Gustavo.TESIS_GRF _210109.pdf

Romero y Suarez (2014) "Evaluación del desempeño docente en una red de colegios particulares de Lima" Red de Colegios Saco Oliveros.

Subaldo L. (2012). Las repercusiones del desempeño docente en la satisfacción y el desgaste del profesorado. Tesis doctoral de la Universidad de Valencia. España.

Toala , S. (2014). Diseño de clima organizacional como mecanismo de atención y su incidencia en el desempeño profesional de los servidores públicos del ilustre Municipio de Jipijapa2013. Trujillo: Tesis doctoral de la Universidad Privada Antenor Orrego.

Valderrama S. (2013). Pasos para elaborar proyectos de investigación científica. Lima: Ed. San Marcos.

Valdés, H. (2004). El desempeño del maestro y su evaluación. La Habana Cuba. Editorial Pueblo y Educación.

Valdés, V. H. (2006). Evaluación del Desempeño docente. Ponencia presentada por Cuba. México: Encuentro Iberoamericano sobre Evaluación del Desempeño docente. Recuperado de http://www.campusoei.org/de/rifad01.htm 